\section{Boosting cell signalling}

The PIM family of serine/threonine kinases consists of at least three members that can cooperate with themyc or bcl-2 oncogenes to induce the development of lymphoid malignancies. To date, however, the precisefunction and physiological substrates of PIM-1 havenot been well characterized. Now, reporting in the Journal of Immunology, Koskinen and colleagues show that PIM-1 phosphorylates NFAT C1 (nuclear factor of activated T cells, cytoplasmic, calcineurin-dependent 1) and enhances NFAT-dependent transcription.

PIM - 1 expression is upregulated during $T$-cell activation. When $T$ cells are activated, AP- 1 (induced by protein kinase $C$ and Ras) and calcium-dependent signalling cause the dephosphorylation of NFATC, which translocates to the nucleus and, together with AP- 1 , activates genes such as interleukin-2 (IL-2).
The authorsfirst looked at therole of PIM -1 kinasein signalling for NFAT activation. Jurkat T cells were transfected with a PIM - 1 expression vector and a reporter construct containing N FAT binding sites. When the T cells were activated with phorbol 12-myristate13-acetate and the calcium ionophore ionomycin, endogenous NFAT activity was enchanced by PIM -1 . To assess whether PIM - 1 targets N FATC in a physiological setting, Jurkat T cellswerestimulated and the amount of IL-2 secreted into the medium was measured. PIM - 1 transfection of stimulated Jurkat cells significantly enhanced IL-2 production compared with non-transfected cells. Kinase-deficient mutants of PIM - 1 were unable to enhance NFAT activity or IL-2 production. Co-immunoprecipitation experimentsshowed that PIM - 1 can physically interact with NFAT C1 and is likely to beableto phosphorylateit in vivo.

This study provides the first evidence that a kinase can positively regulate NFAT activity phosphorylation by other kinases inhibits NFATc translocation to the nucleus. The results suggest that PIM - 1 acts downstream of Ras to induce phosphorylation of N FAT Cl and IL-2 production in Jurkat T cells.

ElaineBell

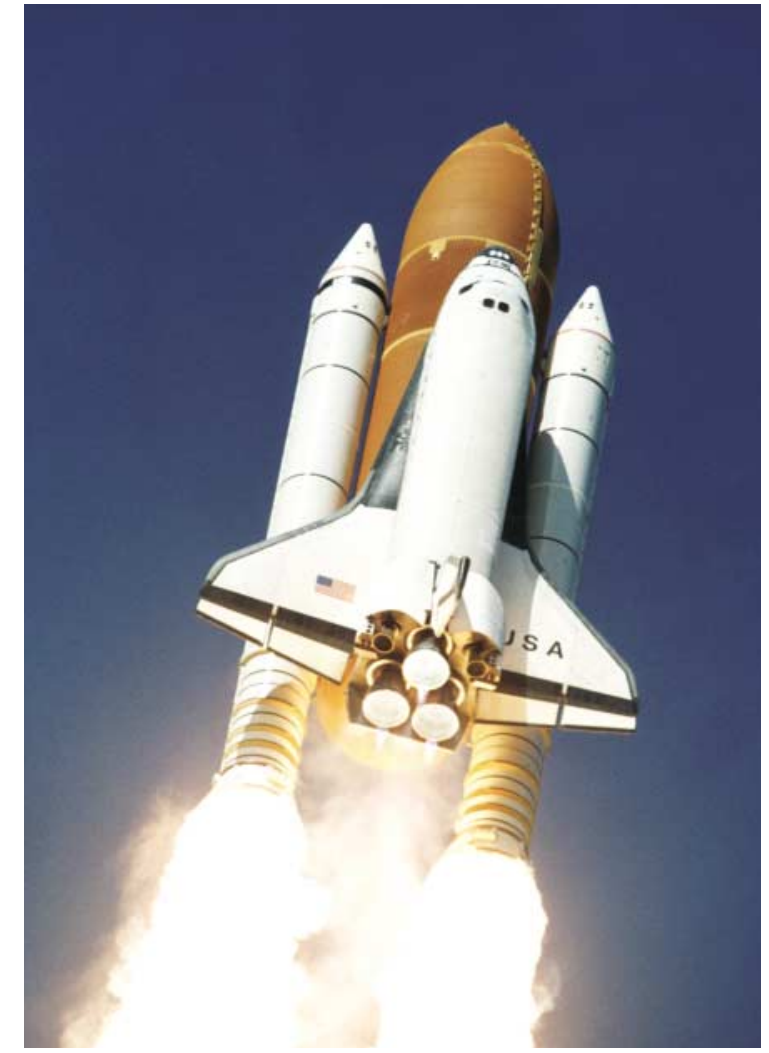

6) References and links

ORIGINAL RESEARCH PAPER Rainio, E-M., Sandholm, J \& \& Koskinen, P. J . Transcriptional activity of NFATC1 is enhanced by the Pim-1 kinase. J . Immunol. 168, 1524-1527 (2002). WEB SITE

Päivi Koskinen's lab: http://www.utu.fi/research/ receptor/projects/koskinen/koskinen.html

peptide conjugated to two fluorophores, but the close proximity of the fluorophores quenches fluorescence. When the substrates are cleaved by active cell caspases the products fluoresce and so the condemned target cell can be detected by flow cytometry and microscopy.

But how does the sensitivity of the new CTL assay compare with the ${ }^{51} \mathrm{Cr}$-release assay? The authors performed the two assays in parallel using splenocytes from mice infected with lymphocytic choriomeningitis virus (LCMV) as effectors and targets pulsed with various LCMV peptides. Thefluorescence based assay was shown to be more sensitive than the ${ }^{51} \mathrm{Cr}$-release assay, particularly at earlier time points.

A key advantage of thenew assay is that the interaction between the target and CTL leading to the delivery of the lethal hit can be visualized directly, in real time, by fluorescence microscopy. In combination with peptide-MHC tetramer staining, this powerful new technique should lead to new insights into the killing process, as well as moreaccurate measurements of CTL responses.

Jennifer Bell

(9) References and links

ORIGINAL RESEARCH PAPER Liu, L. et al. Visualization and quantification of $\mathrm{T}$ cell mediated cytotoxicity using cellpermeable fluorogenic caspase substrates. Nat. Med. 8 , 185-189 (2002). 